\title{
Guest Room Comprehensive Information Management System
}

\author{
Feng Luan \\ Experiment Management Center, Bohai University, Jinzhou, P.R. China \\ 719634434@qq.com
}

Keywords: guest room; comprehensive information; management system; requirement analysis; function design; software architecture

\begin{abstract}
With the rapid development of market economy and the improvement of people's living standards, gaestgiveriet hotel industry is becoming more and more competitive, information system is an important means to improve the level of management and service. This paper follows the idea and method of software engineering to design guest room information management system. Firstly, use the data flow graph technology to carry on the demand analysis, describe the system work process; then, use the tree structure diagram to express the system function, and describe the function module; finally, design by "presentation layer Application, layer Business, logic layer Data, access layer and Data persistence layer Data" and other 5 layers of software architecture. The research content of this paper solve the key technical problems of software development, is the basis for the development and implementation of the system.
\end{abstract}

\section{Introduction}

With the development of information technology, especially the development of database, computer technology has entered into every field of social life and plays a more and more important role. Especially in information management, it has the advantages of convenient, high reliability, large storage capacity, good confidentiality, long life, low cost rather then incomparable advantages of manual management and so on. With the rapid development of market economy and the improvement of people's living standards, gaestgiveriet hotel industry is becoming more and more competitive. Traditional manual management or semi-automated computer management, data redundancy is high, can't be shared, can't achieve financial business data integration, can't provide customers with a full range of information services. For the gaestgiveriet hotel industry, in order to obtain the advantage in the competition, we must improve the service management consciousness in terms of management, product service and so on. Service quality is the key factor to attract customers and increase economic benefit. How to use advanced management means to improve the management level of the guest room is an important issue for every hotel manager. More and more gaestgiveriet hotel use management information system to manage the daily work, reasonable allocate of resources, improve the management level, so as to gain the advantage in the market competition [1,2]. Software design is the basis of system development, this paper is based on the comprehensive system research data, follow by the idea and method of software engineering, use data flow diagram, structure diagram and other tools to design, develop a comprehensive information system to provide technical support.

\section{Requirement Analysis of System}

Requirement analysis describes the purpose, scope, definition, and function of the new system when builds a new or changing existing software system. Requirement analysis is a key process in software engineering, system analyst and the software engineer determine the user's needs. Only after determine the user needs, be able to analyze and seek solutions to the new system. The main task of the requirement analysis phase is to determine the function of the software system. The most common tool for requirement analysis is data flow graph.

Data flow graph is a graphical technique, which describes the transformation of information flow and data from input to output. Data flow graph is the logical model of the system, and there are no 
physical elements in the graph, only describes the flow and processing of information in the system. Because the data flow diagram is a graphical representation of the logic system, even if it is not a professional computer technology personnel, it is an excellent communication tool[3,4].The analyst describes the understanding of the existing system or the idea of the target system with data flow diagrams, for the relevant personnel review and confirm. The data flow diagram of the guest room comprehensive information management system is shown in Fig. 1.

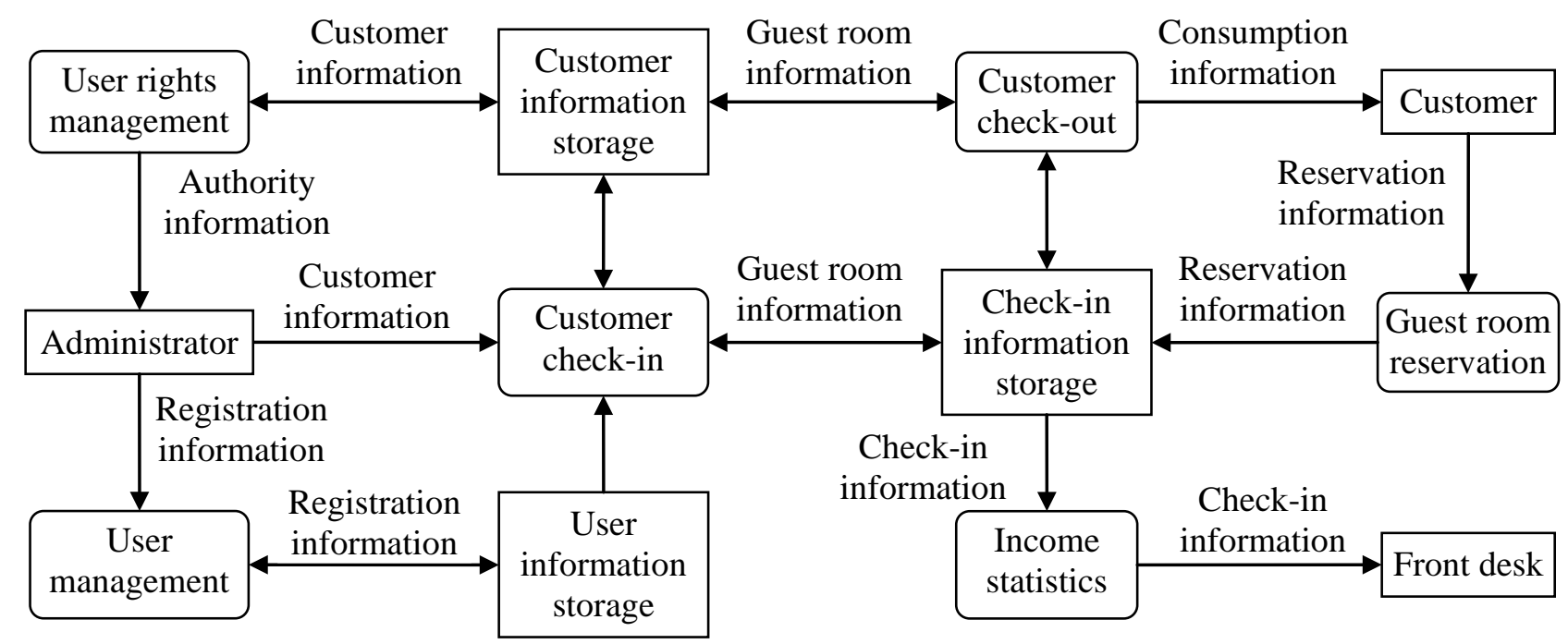

Fig. 1. Data flow chart on guest room comprehensive information management system

According to the data flow diagram show in Fig. 1, a brief description of the process of the system is as follows: the system's users include system administrator, front desk clerk and customer. Customers can be registered in the online remote booking, can also be directly to the front desk for booking. For booking customers, forward to provide information, the reception room information, according to the room information and reservation request to the customer to arrange booking room, and ensure that the room can be delivered to customers on time, customers in accordance with the booking time to stay. Without an appointment of the customer, forward to provide real and effective information to apply for admission, the front desk through the system to check the room information, in line with the customer needs of the room, for customers to check in. When Customers during their stay, the system automatically updates the consumption amount, when the payment isn't enough to pay for the next time period, the system will send fee reminders, alert by the waiter inform customer renewals. When customers leave, customers go to the front desk, and provide the corresponding occupancy information, the reception of customers in the number of days, as well as the identity verification, through the settlement module to check the amount of customers paid, and in accordance with the relevant rules for customers to pay a fee, according to the need to issue payment vouchers and invoices, the system automatically updates customer information and hotel day income information for query statistics. System administrator complete the basic information of the system maintenance and related settings.

\section{Function Design of System}

Effectiveness and function The function of the software is the effectiveness and the function of the software, and the software object must be expressed and realized through the function of the software, software function is also a direct result of the software that present to the user. Users through the function of the software to understand, use and evaluation of the system, through the use of software to complete business functions. Function design is the most important content of software design. Functional design is based on the goal of the software, formed the functional model of the software to describe the results, and quantitative or qualitative describe of the functional requirements of the software. Functional design usually use modular system architecture. Modules are units that can be combined, decomposed and replaced. Modular is a way of dealing 
with complex systems to be better managed. By setting different functions in different components, a problem is decomposed into many small independent and interacting components. The module structure of the functional design of the room integrated information management system is shown in Fig. 2.

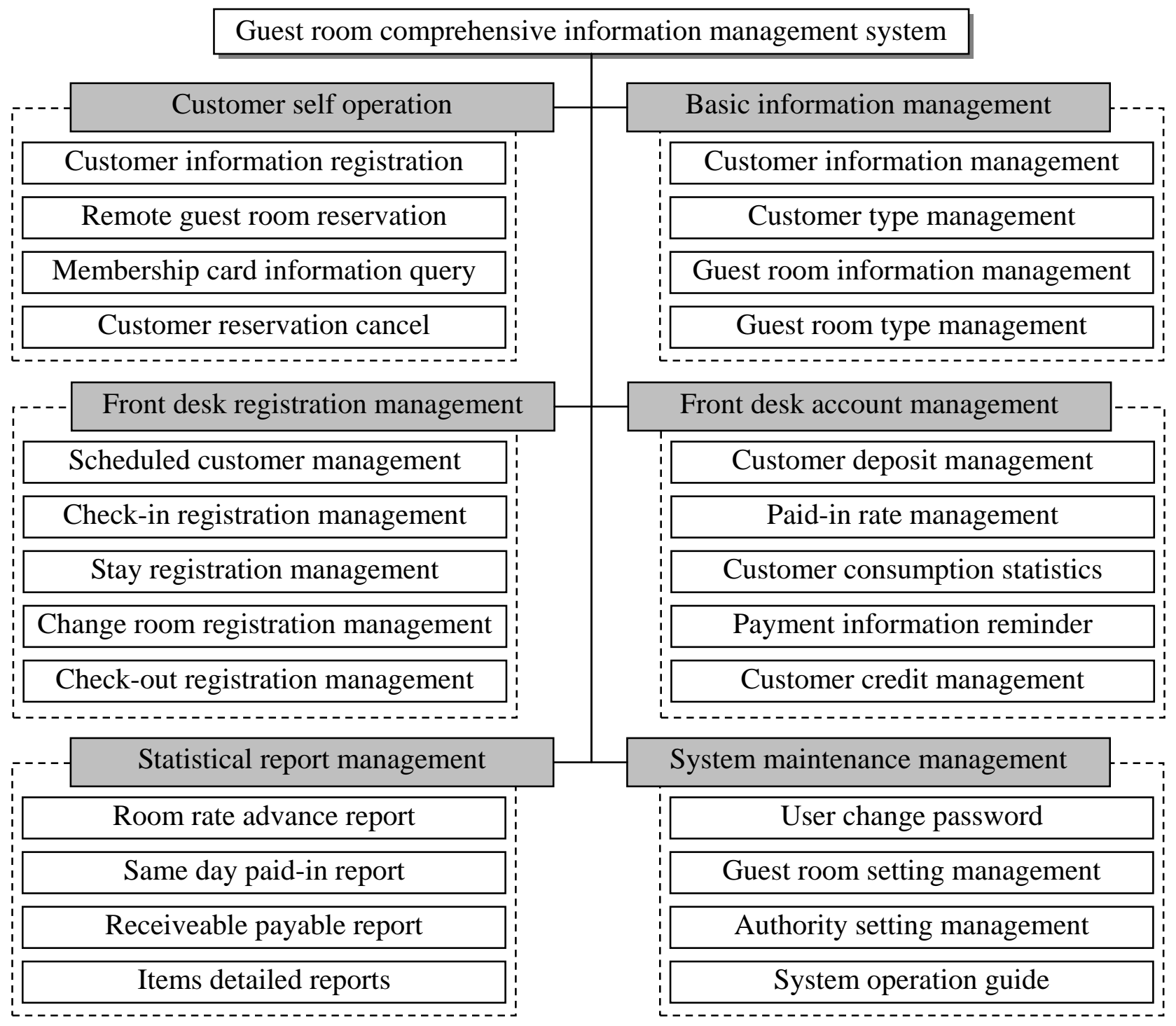

Fig. 2. Modular structure on guest room comprehensive information management system

Fig. 2 includes 6 main functions, each containing a number of sub functions, and a brief description as follow [5-7]: Customer, which provides the operating function for the customer. Customer first register, after the success of the registration can be achieved online customers booking, after the arrival of the customer directly check in procedures. If the customer can't stay on time, should be taken to the message which schedule to make a good reputation; Basic information management, mainly for customers and the relevant information of the room enter, modify, delete and other operations; Front desk registration management, provides the functions of the guest room desk. After the arrival of the customer for the customer check-in, for continued stay, wards, check out operation for the customer has been admitted. Desk account management Front is a function of the room desk operator to cancel the reservation information for the remote client to make room allocation. To check the customer charge pressure gold, for customer billing charge room, for occupancy in arrears customers prompt payment information, for some units provide operating losses; statistical report management, mainly to complete charge of statistics, for the leadership of the view or shift. Including "Room rate advance report, Same day paid-in report, Receiveable payable report, Items detailed reports" and other reports. Maintenance management System, is the operation of the system administrator. System maintenance task is to correct the implicit error found 
in the use of the software system, and expand the new function and performance requirements of the users in the process of using. The purpose is to maintain the normal operation of the software system.

\section{Software Architecture of Software}

Software architecture is a series of related abstract models, which is used to guide the design of large software systems. The software architecture is a system of sketches, which describes the object directly to constitute a system of abstract components. The connection between the various components is clear and relatively detailed description of the communication between the components. In the implementation phase, these abstract components are refined into practical components. In object-oriented domain, the connection between components is usually implemented by the interface. Software architecture design should achieve the following objectives: reliability, software system for the user's business and management is very important, so the software system must be very reliable [8]; security, software system of commercial value, system security is important; customization, the same set of software, can be based on customer base and market demand changes, a software system should allow the introduction of new technology, so that the existing system for power and performance of the system. An easy to maintain system can effectively reduce the cost of technical support; market opportunity, software users to face competition, software providers also face the same industry competition, to the fastest speed for market opportunities is very important. The software architecture of the guest room comprehensive information management system is composed of 5 layers, such as data presentation layer, application layer, business logic layer, data access layer and data persistence layer, as show in Fig. 3.

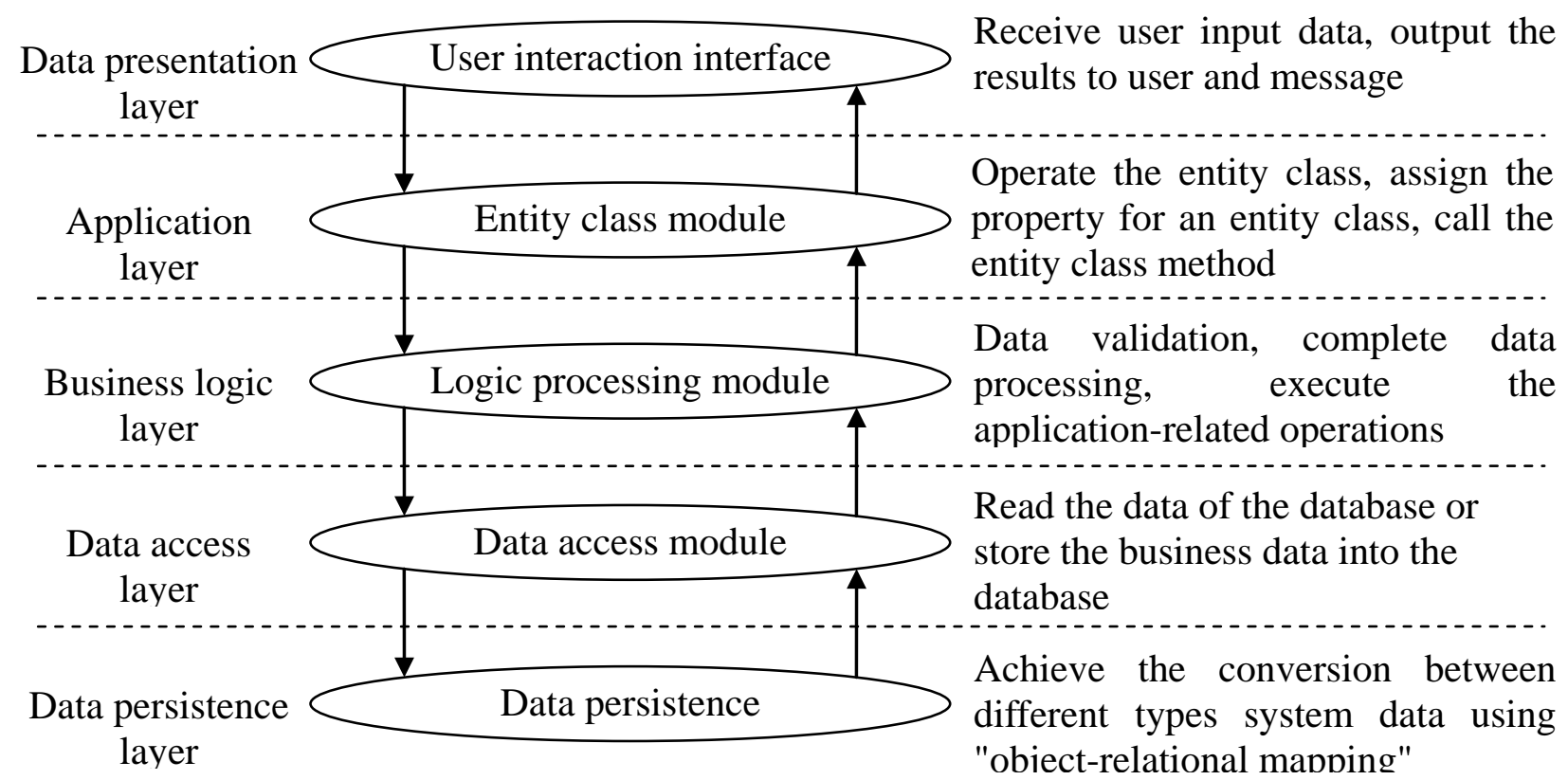

Fig. 3. Software architecture

Data presentation layer. Data presentation layer locates in the outermost layer, is known as the interface layer, responsible for providing users with interactive interface. Provide service to the user, provide the interface, receive the user input, call the business functions, complete the user needs and return the results to the user. At this level, there is no business logic processing, only to call the service logic layer. For example, when the user access interface, can see the input box, data list, pictures, navigation bar, pie chart, etc., are in the presentation layer to complete the show.

Application Layer. Application get the data from data layer to format and calculate the processing, data type conversion, data processing, etc., after the data are transferred to the data of business logic processing layer, business logic layer return data after processing, data type to change, and again the data return to the data presentation layer. 
Business logic layer. A business logic may include a number of inquiries, insert, update, delete, and so on. After the application layer of the program will get the data will create a business all the need to insert, query, delete, update, and other business operations, the operation instructions to the data access layer, data access layer from the data returned to the application layer.

Data access layer. In the business logic layer, accept the data to add, delete, modify, and so on, and then through the general database access module to operate the database, the data return from the database, and then return to the business logic layer.

Data persistence layer. The design objective of the data persistence layer is to provide a high level, unified, secure and concurrent data persistence mechanism for the whole project. To complete the programming of various data, and provide services for the business logic layer. Reasonable data persistence layer and design can simplify the development process of data increase, delete, change, search and other functions, but also not lose the advantages of multi tier structure, inheritance and continuation of the multilayer structure can be scalability and extensibility.

\section{Conclusion}

How to use the advanced management method, improve the hotel management level, has become a top priority for the development of the hotel industry. In the face of the opportunities and challenges in the information age, it is an effective way to improve the management quality by means of scientific and technological means. In this paper, the author designs the general information management system of the hotel rooms, which is based on the actual business of the hotel rooms, according to the research results of this paper, designs guest room comprehensive information management system, achieves the core module of the key modules, such as reservation, customer check, customer checkout, information management and income statistics, and so on. According to the research results of this paper that design room information management system ,achieve the standardization of the management of the room, make customers more fast and efficient to check in, improve the work efficiency, improve the service quality, provide customers with online inquiry and booking services, so that customers can get more personalized service.

\section{References}

[1] Y. Zuang, "The design and implementation of small and medium sized hotel room management system based on Web," Journal of Chifeng University (Natural Science Edition), vol. 31, no. 7, pp. 46-47, 2017.

[2] L. Jiang, "Analysis and design of hotel room management system," Master's degree of Yunnan University, 2013.

[3] H. H. Chen, W. J. Wang, "Design of Visual Edit Tool for DFD Based on UML," Computer Technology and Development, vol. 22, no. 5, pp. 145-149, 2012.

[4] G. J. Fan, Y. E. Hou, Q. Zhang, "The method to construct E-R model of directed graph based on data flow diagram," Computer and Modernization, vol. 30, no. 6, pp. 13-16, 2014.

[5] X. J. Guo, "The design and implementation of hotel room management system based on C\#," China Computer \& Communication, vol. 9, no. 17, pp. 84-85, 2015.

[6] D. H. Duan, "Research and analysis of the hotel room management system," Master's degree of Yunnan University, 2015.

[7] C. H. Wang, J. Q. Cao, "The design and implementation of the hotel room management system for the term project," University Education, vol. 3, no. 3, pp. 71-73, 2014.

[8] Q. R. Li, W. L. Wang, "Design and Realization of Layed Deployment Software Architecture," Intelligent Computer and Applications, vol. 4, no. 2, pp. 45-48, 2014. 